\title{
Mood improvement in young adult males following supplementation with gold kiwifruit, a high-vitamin C food
}

\author{
Anitra C. Carr*, Stephanie M. Bozonet, Juliet M. Pullar and Margreet C. M. Vissers \\ Centre for Free Radical Research, Department of Pathology, University of Otago, Christchurch, PO Box 4345, Christchurch 8140, New Zealand
}

(Received 4 September 2012 - Final revision received 15 April 2013 - Accepted 19 April 2013)

Journal of Nutritional Science (2013), vol. 2, e24, page 1 of 8

doi:10.1017/jns.2013.12

Abstract

Enhanced intakes of fruit and vegetables have been associated with improved psychological well-being. We investigated the potential mood-enhancing effects of kiwifruit, a fruit rich in vitamin $\mathrm{C}$ and a number of other important micronutrients. Young adult males $(n$ 35) were supplemented with either half or two kiwifruit/d for 6 weeks. Profile of Mood States questionnaires were completed at baseline and following the intervention. No effect on overall mood was observed in the half a kiwifruit/d group; however, a $35 \%(P=0.06)$ trend towards a decrease in total mood disturbance and a $32 \%(P=0.063)$ trend towards a decrease in depression were observed in the two kiwifruit/d group. Subgroup analysis indicated that participants with higher baseline mood disturbance exhibited a significant $38 \%(P=0.029)$ decrease in total mood disturbance, as well as a $38 \%(P=0 \cdot 048)$ decrease in fatigue, $31 \%(P=0 \cdot 024)$ increase in vigour and a $34 \%(P=0.075)$ trend towards a decrease in depression, following supplementation with two kiwifruit/d. There was no effect of two kiwifruit/d on the mood scores of participants with lower baseline mood disturbance. Dietary intakes and body status of specific micronutrients indicated a significant increase in the participants' vitamin $\mathrm{C}$ intakes and corresponding plasma levels of the vitamin. The results indicate that enhanced intake of kiwifruit by individuals with moderate mood disturbance can improve overall mood.

Key words: Ascorbate: Plasma vitamin C: Mood disturbance: Dietary intake

The human brain and nervous system have an absolute requirement for specific dietary nutrients such as essential fatty acids, amino acids, vitamins and minerals for proper physiological and psychological functioning ${ }^{(1)}$. Deficiency of specific micronutrients can result in changes in mental function that precede overt deficiency diseases. For example, the symptoms of depression, fatigue and irritability are known to precede the overt physical symptoms of the vitamin C deficiency disease scurvy ${ }^{(2,3)}$. Individuals afflicted with various mental disorders, such as attention deficit-hyperactivity disorder and bipolar disorder, as well as stress, have shown significant improvement in their symptoms, including improved or stabilised mood, following supplementation with multivitamin and mineral formulas ${ }^{(4-6)}$. Even apparently healthy individuals exhibited improved mental functioning and mood following supplementation with these formulas ${ }^{(7-10)}$. Thus, it is likely that mood symptoms are associated with nutrient inadequacy.

Fruit and vegetables are rich in numerous essential vitamins and minerals, particularly vitamin $\mathrm{C}$ and carotenoids ${ }^{(11)}$. Recent research has shown that increased consumption of fruit and vegetables is associated with enhanced psychological well-being ${ }^{(12-14)}$ and decreased depression ${ }^{(15)}$. Kiwifruit are an outstanding source of vitamin $\mathrm{C}^{(16)}$, and also contain reasonable levels of other important micronutrients such as vitamins $\mathrm{B}, \mathrm{E}$ and $\mathrm{K}$, carotenoids and the minerals $\mathrm{Cu}$ and $\mathrm{Mg}^{(17)}$. Since many of these micronutrients have been associated with improved mood ${ }^{(18-21)}$, it is possible that increased ingestion of kiwifruit might enhance mood, particularly in individuals with low daily fruit and vegetable intakes.

Abbreviations: high dose, two kiwifruit/d; low dose, half a kiwifruit/d; POMS, Profile of Mood States; TMD, total mood disturbance.

* Corresponding author: Dr Anitra Carr, fax +643378 6540, email anitra.carr@otago.ac.nz

(C) The Author(s) 2013. The online version of this article is published within an Open Access environment subject to the conditions of the Creative Commons Attribution-NonCommercial-ShareAlike licence <http://creativecommons.org/licenses/by-nc-sa/2.5/>. The written permission of 
The aim of the present study was to investigate the effect of daily supplementation with kiwifruit (Actinidia chinensis var. Hort16A) on the subjective mood of young adult males. We have previously shown that consumption of half a kiwifruit/ $\mathrm{d}$ results in a significant increase in plasma vitamin $\mathrm{C}$ in individuals with low fruit and vegetable intakes, while two kiwifruit/d are required to saturate the plasma, as indicated by a significant increase in urinary vitamin $\mathrm{C}$ excretion at this dose ${ }^{(22)}$. Therefore, in the present study we compared the effect of a low-dose intervention (half a kiwifruit/d) and a high-dose intervention (two kiwifruit/d) on subjective mood in our participants.

We used a Profile of Mood States (POMS) questionnaire to assess the effect of kiwifruit intervention. This is a wellvalidated test useful for monitoring responses to short-term therapeutic interventions and which also has normative data for young adult males ${ }^{(23)}$. In order to elucidate which micronutrients might be playing a role in mood improvement, dietary intakes and circulating and tissue levels of specific micronutrients were measured, particularly those found in kiwifruit, for example, vitamins $\mathrm{C}$ and $\mathrm{E}$ and carotenoids ${ }^{(17)}$, and those known to be influenced by kiwifruit consumption, for example, $\mathrm{Fe}^{(24)}$. Vitamin $\mathrm{D}$ has a putative influence on $\operatorname{mood}^{(25)}$, and because it is obtained primarily through exposure to sunlight rather than through the diet, circulating 25-hydroxyvitamin $\mathrm{D}_{3}$ levels were also monitored.

\section{Method}

\section{Participants}

The present study was conducted according to the guidelines laid down in the Declaration of Helsinki and all procedures involving human participants were approved by the Upper South Regional Ethics Committee (no. URA/11/02/003). The study was registered with the Australian New Zealand Clinical Trials Registry (no. ACTRN12611000162910).

A total of 134 non-smoking male tertiary-level students (aged 18-35 years) were screened for the present study. Exclusion criteria included recent smoker (within previous year), allergy/intolerance to kiwifruit, high plasma vitamin $\mathrm{C}$ levels (>50 $\mu \mathrm{mol} / \mathrm{l})$, taking vitamin C-containing supplements (within past 3 months), taking prescription medication (within past 3 months), excessive alcohol consumption ( $>21$ standard drinks/week), high fruit and vegetable consumption $(>5$ servings/d), diabetes mellitus, bleeding disorders, and fainting due to fear of needles. Anthropometric measurements were carried out to determine BMI, fasting blood samples were drawn to determine circulating vitamin $\mathrm{C}$ levels and skin carotenoid scores were determined non-invasively (see below).

Sample size calculations indicated that at $80 \%$ power and $\alpha=0 \cdot 05$, a sample size of fifteen participants per intervention group would detect a minimum difference of $10 \mu \mathrm{mol} / 1$ ascorbate as determined using data derived from our vitamin $\mathrm{C}$ bioavailability study ${ }^{(22)}$. Using a sample size of fifteen subjects, Zhang et al. ${ }^{(26)}$ observed a significant vitamin C-dependent 10-unit change in total mood disturbance (TMD), as determined using the POMS questionnaire. To allow for potential withdrawal due to the length of the study, thirty-six of the screened individuals who met the inclusion criteria, including below-average plasma vitamin C status, were enrolled in the study. Informed consent was obtained from the participants.

Anthropometric measurements of the enrolled individuals indicated a mean age of 21 (SD 3) years, weight of 85 (SD 20) $\mathrm{kg}$, height of 181 (SD 7) $\mathrm{cm}$ and BMI of 26 (SD 5) $\mathrm{kg} / \mathrm{m}^{2}$. Fasting venous blood samples and skin scans of the enrolled individuals gave a mean plasma vitamin $C$ level of 34 (SD 9) $\mu \mathrm{mol} / 1$ and skin carotenoid score of $24(\mathrm{SD} 7) \times$ $10^{3}$ units. There were no differences between the characteristics of the two intervention groups with respect to age, weight, height, BMI, plasma vitamin $\mathrm{C}$ or skin carotenoid status. Of the participants, one in the high-dose arm withdrew before the beginning of the study.

\section{Study design}

The study employed a parallel-arms design and the participants were randomised into a low-dose group (half a kiwifruit/d) or a high-dose group (two kiwifruit/d) using a random numbers chart. The study comprised a lead-in phase of 5 weeks to allow the participants time to regulate their fruit and vegetable intake, particularly substituting high-vitamin $\mathrm{C}$ content foods with low-vitamin $\mathrm{C}$ content foods, and eliminating juice and cordial from their diet. Baseline values were determined at the end of this 5-week lead-in period. The intervention phase consisted of 6 weeks' supplementation with half or two kiwifruit/d. The participants completed a POMS questionnaire at baseline and following the 6-week kiwifruit intervention. The participants also completed a $7 \mathrm{~d}$ food and drink record at baseline and following the intervention, to monitor their intake of fruit and vegetables as well as dietary intakes of specific micronutrients. Fasting venous blood samples were drawn at baseline and following the intervention to monitor the participants' circulating levels of vitamins $C$ and $\mathrm{D}$ and ferritin. Skin carotenoid levels were measured noninvasively at baseline and following intervention (see below).

\section{Intervention}

Gold kiwifruit (A. chinensis var. Hort16A) were provided by Zespri International Ltd, Mount Maunganui, New Zealand, and were stored at $\leq 4^{\circ} \mathrm{C}$. Participants were provided with kiwifruit each week and were asked to consume half or two kiwifruit/d. The vitamin $\mathrm{C}$ content of the kiwifruit as monitored by HPLC with electrochemical detection ${ }^{(22)}$ was 116 (SD 9) $\mathrm{mg} / 100 \mathrm{~g}$ fruit ( $n$ 5). The participants were asked not to consume the skin, and based on the actual amount of fruit ingested, the amount of vitamin $\mathrm{C}$ consumed was calculated to be about $53 \mathrm{mg}$ per half kiwifruit or about $212 \mathrm{mg}$ for two kiwifruit.

\section{Profile of Mood States questionnaires}

The POMS questionnaire comprises sixty-five mood-related adjectives rated on a five-point Likert-type scale ranging from 0 (not at all) to 4 (extremely) and these are allocated to six clearly defined factors (with maximum scores indicated): 
tension-anxiety (36); depression-dejection (60); anger-hostility (48); vigour-activity (32); fatigue-inertia (28); confusionbewilderment (28). A TMD score can be derived from these (maximum score 200). Normative data for male college students have been reported ${ }^{(23)}$. The participants completed a POMS standard form at baseline and following 6 weeks of intervention. Their tension, depression, anger, fatigue, confusion, vigour and TMD scores were calculated using a POMS standard scoring grid (Psychological Assessments Australia).

\section{Analysis of food and beverage records}

The participants recorded their daily food and beverage intake for 1 week both pre- and post-intervention as described previously ${ }^{(22)}$. The vitamins $\mathrm{C}$ and $\mathrm{E}$ and $\beta$-carotene content of the foods and beverages consumed by the participants were estimated using Diet Cruncher software (version 1.6; Way Down South Software) and the New Zealand FOOD files Food Composition Database (2006).

\section{Sample analysis}

Plasma and urinary ascorbate. Peripheral blood was collected into $\mathrm{K}_{3}$-EDTA vacutainer tubes and kept on ice at all times. Plasma was collected following centrifugation for extraction of ascorbate. Urine was collected over $24 \mathrm{~h}$ into pre-weighed bottles containing $\mathrm{K}_{2}$-EDTA (final concentration $100 \mu \mathrm{mol} / \mathrm{l})$. The plasma and urine samples were deproteinated by adding an equal volume of ice-cold 0.54 M-perchloric acid containing diethylenetriaminepentaacetic acid (DTPA) $(100 \mu \mathrm{mol} / \mathrm{l})$ followed by vortex mixing and centrifugation $^{(22)}$ and the samples were stored at $-80^{\circ} \mathrm{C}$. Ascorbate content was analysed using reverse-phase HPLC with electrochemical detection as described previously ${ }^{(22)}$.

Serum ferritin. Serum derived from peripheral blood samples was analysed for ferritin content by Canterbury Health Laboratories using a chemiluminescent microparticle immunoassay (Abbott Architect i2000).

Serum 25-hydroxyvitamin D. Serum derived from peripheral blood samples was analysed for 25-hydroxyvitamin $\mathrm{D}_{3}$ content by HPLC-MS/MS using a Shimadzu 20 series HPLC system with a C8 reverse-phase column coupled to an ABI 4000 mass spectrometer as described previously ${ }^{(27)}$.

Skin carotenoids. Skin carotenoids were measured noninvasively using a Pharmanex Biophotonic Scanner (Nuskin). The scanner utilises a low-energy blue light laser (which emits at $473 \mathrm{~nm}$ and receives at $510 \mathrm{~nm}$ ) and operates on the principles of resonance Raman spectroscopy ${ }^{(28)}$.

\section{Statistical analysis}

Data are represented as either mean values and standard deviations or mean values with their standard errors, as indicated in the text. The differences between paired and unpaired data were determined by two-tailed $t$ test and $P$ values $\leq 0.05$ were considered statistically significant.

\section{Results}

\section{Profile of Mood States}

The participants completed POMS questionnaires at baseline and following 6 weeks' supplementation with either half or two kiwifruit/d. Analysis of the individual mood and TMD scores of the participants indicated no effect of intervention with half a kiwifruit/d (Fig. 1(a)). In contrast, participants supplemented with two kiwifruit/d showed a $35 \%$ decrease in TMD score, which was trending towards significance $(P=0.061$; Fig. 1(b)). A similar trend ( $32 \%$ decrease) was also observed for the depression score with two kiwifruit/d ( $P=0.063$; Fig. 1(b)).

The participants' average mood scores were comparable with normative data for male college students ${ }^{(23)}$. Since an intervention effect would probably be more pronounced in individuals with higher baseline mood disturbance, the participants in each of the two intervention groups were separated into subgroups of lower than average and higher than average mood disturbance. This was accomplished by dividing the groups around their mean baseline TMD scores, i.e. 26 and 25 for the half a kiwifruit/d and two kiwifruit/d groups, respectively (Fig. 1). This resulted in the high-TMD subgroups having average scores eight- to ten-fold higher than the mean of the low-TMD subgroups; for example, 48 v. $5(P<0.001)$, for the high- and low-TMD subgroups in the two kiwifruit/d intervention group (Fig. 2).

Following supplementation with half a kiwifruit/d, there was no effect on mood scores in either the low- or high-TMD subgroups (data not shown). However, supplementation with two kiwifruit/d resulted in an improved mood score in the high-TMD subgroup (Fig. 2(a)), with a $38 \%$ decrease in TMD score $(P=0.029)$, a $38 \%$ decrease in fatigue $(P=0.048)$, a $31 \%$ increase in vigour $(P=0.023)$, and a $34 \%$ decrease in depression, which was trending towards significance $(P=0.075)$. In contrast, there was no effect of two kiwifruit/d on the scores of the low-TMD subgroup (Fig. 2(b)). This indicates that the trend towards mood improvement observed in the initial two kiwifruit/d intervention group ( $n$ 15) was probably due to effects on the participants with higher baseline mood disturbance.

\section{Micronutrient intake and status}

At baseline the participants' mean servings of fruit and vegetables were $<3 / \mathrm{d}$ (Table 1$)$. In the low-dose fruit group a non-significant increase to 3.4 servings/d was observed, while the high-dose fruit supplementation resulted in a significant increase to nearly 5 servings of fruit and vegetables/d (Table 1); current recommendations are 5-9 servings/ $\mathrm{d}^{(29)}$.

The participants' mean vitamin $\mathrm{C}$ intake at baseline was $29 \mathrm{mg} / \mathrm{d}$ (Table 1); British and Australasian recommended dietary intakes are 40 and $45 \mathrm{mg} / \mathrm{d}$, respectively. Following 6 weeks of intervention with kiwifruit the vitamin $\mathrm{C}$ intake had increased significantly for both the low- and high-dose 

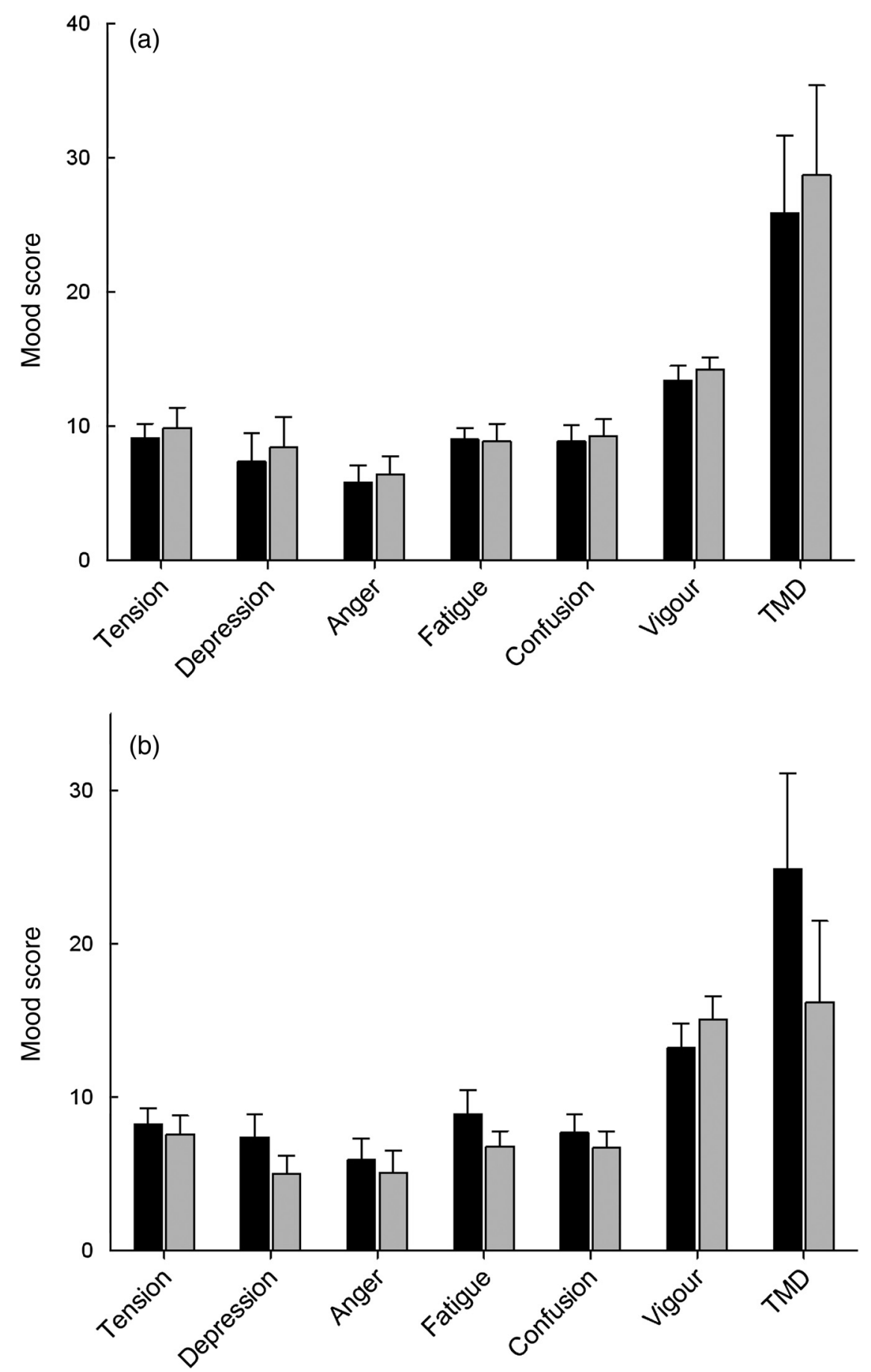

Fig. 1. Individual mood scores and total mood disturbance (TMD) score at baseline ( $\square$ ) and following 6 weeks' intervention ( $\square$ ) with (a) half a kiwifruit/d or (b) two kiwifruit/d. Data are means, with standard errors represented by vertical bars.

groups (Table 1) and this was nearly all accounted for by thekiwifruit intervention (data not shown). The participants' baseline plasma vitamin $\mathrm{C}$ levels of 23 and $25 \mu \mathrm{mol} / 1$ increased to $46 \mu \mathrm{mol} / 1$ for the low-dose group and to 63 $\mu \mathrm{mol} / \mathrm{l}$ for the high-dose group $(P<0 \cdot 001$; Table 1). Following intervention, there was only a very small increase in urinary vitamin $\mathrm{C}$ excretion in the low-dose group, but a large 15-fold increase was seen in the high-dose group (Table 1). This indicates that plasma ascorbate is saturating with the high-dose kiwifruit.
The participants' vitamin E intake also increased significantly in the two kiwifruit/d group but not in the half a kiwifruit/d group, although there was no significant difference between the two groups following intervention (Table 1). There were no significant differences in the participants' $\beta$-carotene intakes following intervention (Table 1). The participants' baseline skin carotenoid scores were 21 and 24 units $\times 10^{3}$ for the low-dose and high-dose fruit groups, respectively. These showed a small drop in the high-dose fruit group $(P<0 \cdot 05)$; however, there were no significant differences between the two intervention 

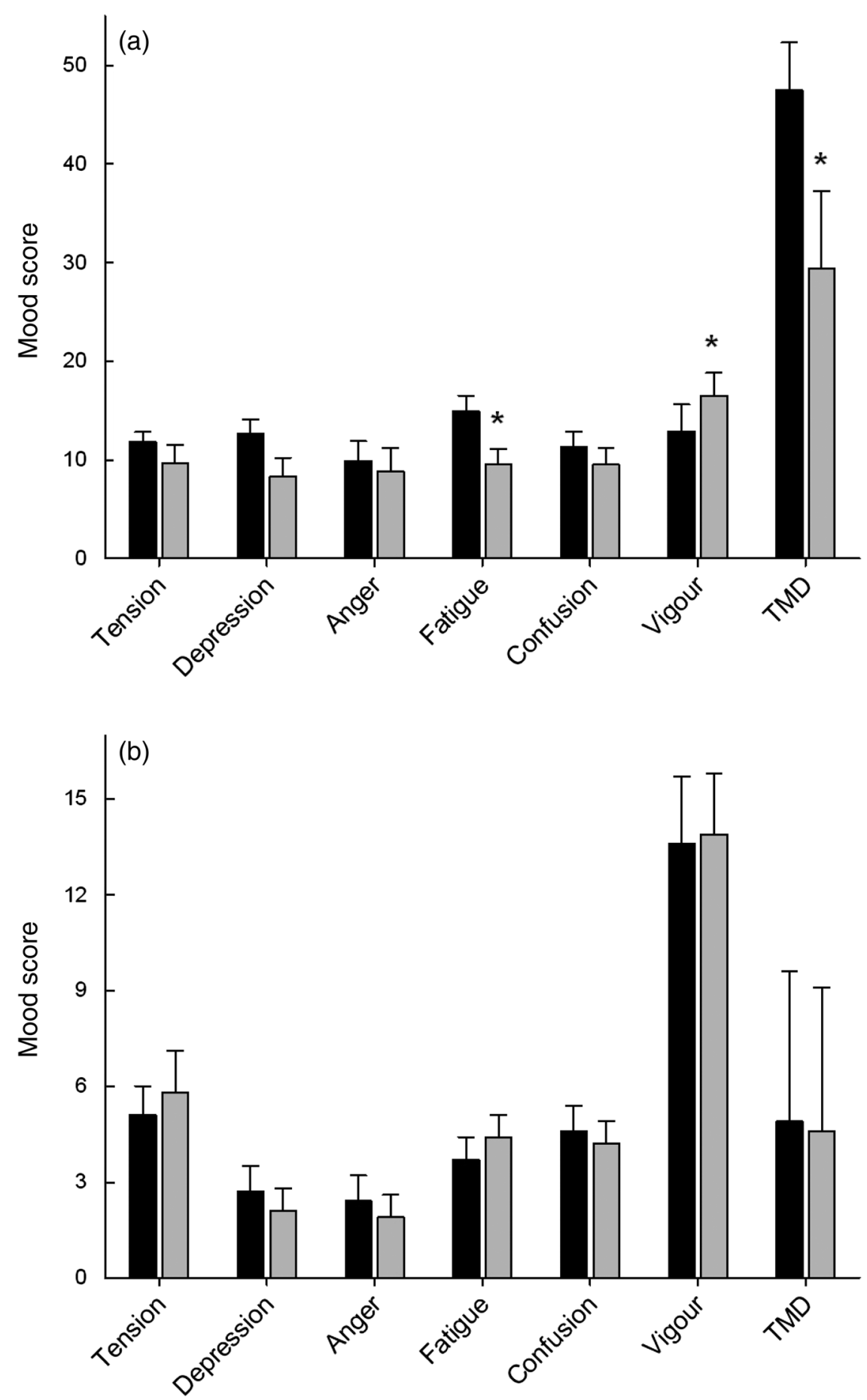

Fig. 2. Effect of two kiwifruit/d intervention on individual mood scores and total mood disturbance (TMD) score in (a) the high-TMD subgroup ( $n$ 8) and (b) the low-TMD subgroup $(n 9)$. $\mathbf{\square}$, Baseline; $\ldots$, intervention. Data are means, with standard errors represented by vertical bars. ${ }^{*}$ Mean value was significantly different from that at baseline $(P<0.05$; two-tailed paired $t$ test).

groups (Table 1). The participants' serum ferritin levels at baseline for the low-dose and high-dose fruit groups were, respectively, 114 and $112 \mu \mathrm{g} / \mathrm{l}$, which were within the normal adult range of $20-500 \mu \mathrm{g} / 1$, and these did not change following intervention (Table 1). The participants' baseline 25-hydroxyvitamin $\mathrm{D}_{3}$ levels for the low-dose and high-dose fruit groups, respectively, were 46 and $40 \mathrm{nmol} / 1$ (optimal range is $50-150 \mathrm{nmol} / \mathrm{l}$ ) and these increased marginally in the high-dose fruit group $(P<$ $0 \cdot 05)$, although there were no significant differences between the two intervention groups (Table 1). Similarly, there were no differences in any of the analysed micronutrients in the highand low-TMD subgroups of the two intervention groups (data not shown).

\section{Discussion}

The present study showed a trend towards a decrease in overall mood disturbance and depression in young adult males 
Table 1. Micronutrient intake and circulating and tissue levels of selected micronutrients pre- and post-intervention with half or two kiwifruit/d (Mean values with their standard errors)

\begin{tabular}{|c|c|c|c|c|c|c|c|c|c|}
\hline & \multicolumn{4}{|c|}{ Half a kiwifruit/d ( $n$ 18) } & \multicolumn{4}{|c|}{ Two kiwifruit/d ( $n$ 17) } & \multirow[b]{3}{*}{ Between-group intervention: $P \dagger$} \\
\hline & \multicolumn{2}{|c|}{ Baseline } & \multicolumn{2}{|c|}{ Intervention } & \multicolumn{2}{|c|}{ Baseline } & \multicolumn{2}{|c|}{ Intervention } & \\
\hline & Mean & SEM & Mean & SEM & Mean & SEM & Mean & SEM & \\
\hline Fruit and vegetable servings (per d) & $2 \cdot 9$ & 0.3 & 3.4 & 0.4 & $2 \cdot 7$ & 0.2 & $4 \cdot 8^{\star \star \star}$ & 0.3 & 0.001 \\
\hline Vitamin C intake (mg/d) & 29 & 3 & $80^{\star \star *}$ & 4 & 29 & 3 & $241^{* \star *}$ & 5 & $<0.0001$ \\
\hline Vitamin $E$ intake (mg/d) & 6.4 & 0.6 & 8.5 & $1 \cdot 1$ & $7 \cdot 0$ & 0.7 & $9 \cdot 9^{\star *}$ & 0.4 & 0.2 \\
\hline$\beta$-Carotene intake $(\mathrm{mg} / \mathrm{d})$ & $2 \cdot 0$ & 0.2 & 1.6 & 0.3 & 1.6 & 0.2 & 1.8 & 0.3 & 0.8 \\
\hline Plasma ascorbate $(\mu \mathrm{mol} / \mathrm{l})$ & 23 & 3 & $46^{\star \star \star}$ & 3 & 25 & 3 & $63^{\star \star \star}$ & 3 & $<0.0001$ \\
\hline Skin carotenoid score $\left(\mathrm{U} \times 10^{3}\right)$ & 21 & 2 & 19 & 2 & 24 & 2 & $21^{*}$ & 2 & 0.3 \\
\hline Serum ferritin $(\mu \mathrm{g} / \mathrm{l})$ & 114 & 13 & 116 & 16 & 112 & 13 & 118 & 19 & 0.9 \\
\hline Serum 25-hydroxyvitamin $\mathrm{D}_{3}(\mathrm{nmol} / \mathrm{l})$ & 46 & 6 & 50 & 7 & 40 & 4 & $46^{*}$ & 4 & 0.5 \\
\hline Urinary ascorbate $(\mu \mathrm{mol} / 24 \mathrm{~h})$ & 32 & 8 & $71^{*}$ & 17 & 32 & 11 & $485^{\star \star *}$ & 70 & $<0.0001$ \\
\hline
\end{tabular}

Mean value was significantly different from that at baseline: ${ }^{*} P<0.05,{ }^{* *} P<0.01,{ }^{* * *} P<0.0001$ (paired two-tailed $t$ test).

† Unpaired $t$ test for half a kiwifruit/d $v$. two kiwifruit/d interventions. There were no significant differences between the baseline levels of the two groups.

consuming two kiwifruit/d for 6 weeks. Further analysis of a subgroup of individuals with higher than average mood disturbance indicated a significant decrease in fatigue, a concomitant increase in vigour, and a trend towards a decrease in depression following supplementation with two kiwifruit/d, but not half a kiwifruit/d, which indicates a possible dose effect. Although a significant increase in plasma vitamin C was observed with the half a kiwifruit/d dose, this level (i.e. $46 \mu \mathrm{mol} / \mathrm{l}$ ) is still considered suboptimal and inadequate for health maintenance ${ }^{(30)}$. The two kiwifruit/d dose resulted in saturating levels of vitamin C (i.e. about $63 \mu \mathrm{mol} / \mathrm{l}$ ). This dose of kiwifruit provided $>200 \mathrm{mg} / \mathrm{d}$ of vitamin $\mathrm{C}$ and is considered an optimal intake ${ }^{(31)}$.

We have previously observed a significant improvement in mood in a depressed individual with hypovitaminosis $\mathrm{C}$ following supplementation with $1000 \mathrm{mg} / \mathrm{d}$ of vitamin $\mathrm{C}^{(19)}$ and other studies have shown reductions in depression ${ }^{(32)}$, fatigue and mood disturbance in cancer patients provided with intravenous vitamin $\mathrm{C}^{(33,34)}$, in obese individuals supplemented with 500 $\mathrm{mg} / \mathrm{d}$ vitamin $\mathrm{C}^{(35)}$, and in critically ill and psychiatric patients supplemented with $1000 \mathrm{mg} / \mathrm{d}$ of vitamin $C^{(26,36,37)}$. Apparently healthy individuals have also benefited from vitamin C supplementation with respect to depression, stress and fatigue $^{(38-41)}$. Fatigue and irritability are common symptoms in individuals with subclinical vitamin $\mathrm{C}$ deficiency and are resolved with vitamin $\mathrm{C}$ supplementation ${ }^{(3)}$. Cheraskin et al. ${ }^{(42)}$ also noted a negative association between vitamin $\mathrm{C}$ intake and fatigue, with a doubling of symptoms observed in individuals consuming $<100 \mathrm{mg} / \mathrm{d}$ vitamin C compared with $>400 \mathrm{mg} / \mathrm{d}$ vitamin $C^{(42)}$. The effect of vitamin $C$ on fatigue could be explained by the role of vitamin $\mathrm{C}$ as a cofactor for the two dioxygenase enzymes involved in the biosynthesis of carnitine, a compound required for generation of metabolic energy ${ }^{(43)}$.

Vitamin C has a number of functions that could potentially affect depression. It has been proposed to function as a neuromodulator of both dopamine- and glutamate-mediated neurotransmission ${ }^{(44)}$. It also acts as a cofactor for the enzyme dopamine $\beta$-hydroxylase that converts dopamine into the neurotransmitter noradrenaline ${ }^{(45)}$. Brain noradrenaline levels are decreased in vitamin C-deficient guinea-pigs ${ }^{(46,47)}$ and depletion is associated with depressive symptoms in humans ${ }^{(48)}$. Vitamin C also serves to activate the enzyme tyrosine hydroxylase via recycling its cofactor tetrahydrobiopterin, which is required for the conversion of $\mathrm{L}$-tyrosine to the dopamine precursor L-DOPA (L-3,4-dihydroxyphenylalanine) ${ }^{(49)}$. Peptide amidation, which occurs during the biosynthesis of various peptide-based hormones, hormone-releasing factors and neurotransmitters ${ }^{(50)}$, also involves a hydroxylating enzyme which has been shown to require vitamin $C^{(51)}$. One of these peptide-based hormones is oxytocin, which has known mood-enhancing effects ${ }^{(52,53)}$.

Although kiwifruit contain very high levels of vitamin C they also contain reasonable levels of other important micronutrients ${ }^{(17)}$ which may contribute to improved $\operatorname{mood}^{(18)}$. Low vitamin E status has been shown to be associated with major depression $^{(54,55)}$ and although our participants' vitamin E intake was significantly higher in the two kiwifruit/d group, there were no significant differences between the two intervention groups and no correlation of vitamin $\mathrm{E}$ intake with mood disturbance (data not shown). However, vitamin $\mathrm{C}$ has been shown to interact with vitamin $\mathrm{E}$ in vivo ${ }^{(56)}$ and combined deficiency of vitamins $\mathrm{C}$ and $\mathrm{E}$ can cause severe central nervous system damage ${ }^{(57)}$. Thus an interaction between vitamins $\mathrm{C}$ and $\mathrm{E}$ is possible.

Low carotenoid status has been associated with depressive disorders ${ }^{(20)}$, while higher carotenoid status is associated with optimism ${ }^{(21)}$. However, these studies report correlations only and carotenoid levels may simply reflect fruit and vegetable intake ${ }^{(11,58)}$. In the present study we did not observe any significant increases in $\beta$-carotene intake or skin carotenoid scores following intervention and no differences between the two intervention groups. However, it should be noted that kiwifruit themselves have a relatively low $\beta$-carotene content, i.e. $<1 \%$ that of carrots ${ }^{(59)}$.

Dietary $\mathrm{Fe}$ uptake is known to be enhanced by kiwifruit ingestion $^{(24)}$, and this mineral has a vital role in brain function and mood ${ }^{(18)}$. The lack of an effect of kiwifruit intervention on ferritin levels in our present study is likely to be due to the participants' baseline $\mathrm{Fe}$ status being already within the normal range as well as a lack of co-supplementation with dietary $\mathrm{Fe}$ 
(kiwifruit themselves contain only very low levels of $\mathrm{Fe}^{(17)}$ ). Vitamin D status has putative associations with mood and depression $^{(25)}$, and although our participants had less than optimal levels of 25-hydroxyvitamin $\mathrm{D}_{3}$ at baseline and showed a small increase in the high-dose intervention group, we did not observe any differences in circulating levels of 25-hydroxyvitamin $\mathrm{D}_{3}$ between the two intervention groups.

When carrying out intervention studies it is critical to recruit participants with the appropriate baseline characteristics. For example, we and others have shown that vitamin $\mathrm{C}$ supplementation studies will not prove effective if the participants are already replete ${ }^{(22,30)}$. In our present study we did not preselect our participants based on their mood disturbance scores and no effect of kiwifruit intervention was observed in individuals who exhibited lower than average mood disturbance symptoms at baseline. The subgroup analysis allowed us to determine whether there was an effect in those individuals with the highest mood disturbance scores. Due to the relatively small sample sizes used in the subgroup analysis $(n 8$ and 9), a group effect cannot be ruled out. However, Huck et al. ${ }^{(35)}$ showed a significant 6-unit vitamin C-dependent decrease in fatigue with a sample size of nine participants using the POMS questionnaire. Nevertheless, further studies would ideally comprise larger numbers of participants. A parallel group of individuals who were supplemented with $50 \mathrm{mg} / \mathrm{d}$ of purified vitamin $\mathrm{C}$, comparable with that found in half a kiwifruit, also did not show any improvement in mood disturbance (data not shown). Therefore, studies supplementing individuals with doses of vitamin $C \geq 200 \mathrm{mg} / \mathrm{d}$ (i.e. comparable with that found in two kiwifruit) appear warranted in order to determine if kiwifruit-derived vitamin $\mathrm{C}$ is the active factor in the present study.

Overall, our kiwifruit supplementation study shows that a positive effect on mood and vigour can be measured in an otherwise well population with suboptimal intakes of fruit and vegetables and vitamin $\mathrm{C}$. Of the micronutrients measured, vitamin $C$ was the only one that increased dramatically and which differed significantly between the intervention groups. Thus, it is conceivable that the vitamin $\mathrm{C}$ component of the kiwifruit is contributing to the observed mood improvement. As such, further studies using larger numbers of participants who exhibit high baseline mood disturbance scores, and with high doses of purified vitamin C, appear warranted. These studies would help to clarify the role of vitamin $\mathrm{C}$ with respect to mood in general and fatigue and depression in particular.

\section{Acknowledgements}

We express our gratitude to the young men who participated in the present study, many of whom showed great dedication and perseverance. We acknowledge Maria Webb and Heather Webb for assistance with recruitment, Wathsala Kumarasinghe and Cecilia Sam for the diet analysis, John Lewis and Peter Elder for vitamin D analysis, Jo Kepple for the use of the Primorus Clinical Trials Unit, and Lynley Drummond for consultation on study design.

Financial support for the present study was provided by the University of Otago, Dunedin, New Zealand, and Zespri
International Ltd, Mount Maunganui, New Zealand. The contributions of each author are as follows: M. C. M. V., A. C. C., study design; A. C. C., S. B., recruitment; A. C. C., study coordination, data analysis and writing manuscript; S. B., M. C. M. V., A. C. C., sample processing; S. B., sample analysis; J. P., S. B., M. C. M. V., editing manuscript. Each author has seen and approved the contents of the manuscript.

There are no conflicts of interest.

\section{References}

1. Bourre JM (2006) Effects of nutrients (in food) on the structure and function of the nervous system: update on dietary requirements for brain. Part 1: micronutrients. J Nutr Health Aging 10, 377-385.

2. Crandon JH, Lund CC \& Dill DB (1940) Experimental human scurvy. N Engl J Med 223, 353-369.

3. Levine M, Conry-Cantilena C, Wang Y, et al. (1996) Vitamin C pharmacokinetics in healthy volunteers: evidence for a recommended dietary allowance. Proc Natl Acad Sci U S A 93, 3704-3709.

4. Rucklidge J, Taylor M \& Whitehead K (2011) Effect of micronutrients on behavior and mood in adults with ADHD: evidence from an 8-week open label trial with natural extension. J Atten Disord 15, 79-91.

5. Kaplan BJ, Simpson JS, Ferre RC, et al. (2001) Effective mood stabilization with a chelated mineral supplement: an open-label trial in bipolar disorder. J Clin Psychiatry 62, 936-944.

6. Schlebusch L, Bosch BA, Polglase G, et al. (2000) A double-blind, placebo-controlled, double-centre study of the effects of an oral multivitamin-mineral combination on stress. S Afr Med J 90, 1216-1223.

7. Kennedy DO, Veasey R, Watson A, et al. (2010) Effects of highdose $\mathrm{B}$ vitamin complex with vitamin $\mathrm{C}$ and minerals on subjective mood and performance in healthy males. Psychopharmacology (Berl) 211, 55-68.

8. Kennedy DO, Veasey RC, Watson AW, et al. (2011) Vitamins and psychological functioning: a mobile phone assessment of the effects of a B vitamin complex, vitamin $\mathrm{C}$ and minerals on cognitive performance and subjective mood and energy. Hum Psychopharmacol 26, 338-347.

9. Carroll D, Ring C, Suter M, et al. (2000) The effects of an oral multivitamin combination with calcium, magnesium, and zinc on psychological well-being in healthy young male volunteers: a double-blind placebo-controlled trial. Psychopharmacology (Berl) 150, 220-225.

10. Benton D, Haller J \& Fordy J (1995) Vitamin supplementation for 1 year improves mood. Neuropsychobiology 32, 98-105.

11. Block G, Norkus E, Hudes M, et al. (2001) Which plasma antioxidants are most related to fruit and vegetable consumption? Am J Epidemiol 154, 1113-1118.

12. Blanchflower DG, Oswald AJ \& Stewart-Brown S (2012) Is psychological well-being linked to the consumption of fruits and vegetables? Soc Indic Res (epublication ahead of print version 11 October 2012).

13. Piqueras JA, Kuhne W, Vera-Villarroel P, et al. (2011) Happiness and health behaviours in Chilean college students: a cross-sectional survey. BMC Public Health 11, 443.

14. White BA, Horwath CC \& Conner TS (2013) Many apples a day keep the blues away - daily experiences of negative and positive affect and food consumption in young adults. Br J Health Psychol (epublication ahead of print version 24 January 2013).

15. Tsai AC, Chang TL \& Chi SH (2012) Frequent consumption of vegetables predicts lower risk of depression in older Taiwanese results of a propsective population-based study. Public Health Nutr 15, 1087-1092.

16. Nishiyama I, Yamashita Y, Yamanaka M, et al. (2004) Varietal difference in vitamin $\mathrm{C}$ content in the fruit of kiwifruit and other Actinidia species. J Agric Food Chem 52, 5472-5475.

17. Lesperance L, et al. (2009) The Concise New Zealand Food Composition Tables, 8th ed. Palmerston North: New Zealand Institute for Plant and Food Research Ltd; Wellington, Ministry of Health. 
18. Kaplan BJ, Crawford SG, Field CJ, et al. (2007) Vitamins, minerals, and mood. Psychol Bull 133, 747-760.

19. Carr AC \& Vissers MCM (2012) Good nutrition matters: hypovitaminosis $\mathrm{C}$ associated with depressed mood and poor wound healing. N Z Med J 125, 107-109.

20. Milaneschi Y, Bandinelli S, Penninx BW, et al. (2012) The relationship between plasma carotenoids and depressive symptoms in older persons. World J Biol Psychiatry 13, 588-598.

21. Boehm JK, Williams DR, Rimm EB, et al. (2013) Association between optimism and serum antioxidants in the midlife in the United States study. Psychosom Med 75, 2-10.

22. Carr AC, Pullar JM, Moran S, et al. (2012) Bioavailability of vitamin $\mathrm{C}$ from kiwifruit in non-smoking males: determination of 'healthy' and 'optimal' intakes. J Nutr Sci 1, e14.

23. McNair DM \& Heuchert JWP (2005) Profile of Mood States Technical Update. North Tonawanda: Multi-Health Systems.

24. Beck K, Conlon CA, Kruger R, et al. (2011) Gold kiwifruit consumed with an iron-fortified breakfast cereal meal improves iron status in women with low iron stores: a 16-week randomised controlled trial. Br J Nutr 105, 101-109.

25. Berk M, Sanders KM, Pasco JA, et al. (2007) Vitamin D deficiency may play a role in depression. Med Hypotheses 69, 1316-1319.

26. Zhang M, Robitaille L, Eintracht $S$, et al. (2011) Vitamin $C$ provision improves mood in acutely hospitalized patients. Nutrition $27,530-533$.

27. Maunsell Z, Wright DJ \& Rainbow SJ (2005) Routine isotopedilution liquid chromatography-tandem mass spectrometry assay for simultaneous measurement of the 25-hydroxy metabolites of vitamins $\mathrm{D}_{2}$ and $\mathrm{D}_{3}$. Clin Chem 51, 1683-1690.

28. Rerksuppaphol S \& Rerksuppaphol L (2006) Effect of fruit and vegetable intake on skin carotenoid detected by non-invasive Raman spectroscopy. J Med Assoc Thai 89, 1206-1212.

29. Guenther PM, Dodd KW, Reedy J, et al. (2006) Most Americans eat much less than recommended amounts of fruits and vegetables. J Am Diet Assoc 106, 1371-1379.

30. Lykkesfeldt J \& Poulsen HE (2010) Is vitamin C supplementation beneficial? Lessons learned from randomised controlled trials. Br J Nutr 103, 1251-1259.

31. Levine M, Padayatty SJ \& Espey MG (2011) Vitamin C: a concentration-function approach yields pharmacology and therapeutic discoveries. Adv Nutr 2, 78-88.

32. Cocchi P, Silenzi M, Calabri G, et al. (1980) Antidepressant effect of vitamin C. Pediatrics 65, 862-863.

33. Vollbracht C, Schneider B, Leendert V, et al. (2011) Intravenous vitamin $\mathrm{C}$ administration improves quality of life in breast cancer patients during chemo-/radiotherapy and aftercare: results of a retrospective, multicentre, epidemiological cohort study in Germany. In Vivo 25, 983-990.

34. Yeom CH, Jung GC \& Song KJ (2007) Changes of terminal cancer patients' health-related quality of life after high dose vitamin $\mathrm{C}$ administration. J Korean Med Sci 22, 7-11.

35. Huck CJ, Johnston CS, Beezhold BL, et al. (2012) Vitamin C status and perception of effort during exercise in obese adults adhering to a calorie-reduced diet. Nutrition 29, 42-45.

36. Evans-Olders R, Eintracht S \& Hoffer LJ (2010) Metabolic origin of hypovitaminosis $\mathrm{C}$ in acutely hospitalized patients. Nutrition 26, 1070-1074

37. Milner G (1963) Ascorbic acid in chronic psychiatric patients - a controlled trial. Br J Psychiatry 109, 294-299.

38. Suh SY, Bae WK, Ahn HY, et al. (2012) Intravenous vitamin C administration reduces fatigue in office workers: a double-blind randomized controlled trial. Nutr J 11, 7.
39. Brody S (2002) High-dose ascorbic acid increases intercourse frequency and improves mood: a randomized controlled clinical trial. Biol Psychiatry 52, 371-374.

40. Brody S, Preut R, Schommer K, et al. (2002) A randomized controlled trial of high dose ascorbic acid for reduction of blood pressure, cortisol, and subjective responses to psychological stress. Psychopharmacology (Berl) 159, 319-324.

41. Kinsman RA \& Hood J (1971) Some behavioral effects of ascorbic acid deficiency. Am J Clin Nutr 24, 455-464.

42. Cheraskin E, Ringsdorf WM Jr \& Medford FH (1976) Daily vitamin C consumption and fatigability. J Am Geriatr Soc 24, 136-137.

43. Rebouche CJ (1991) Ascorbic acid and carnitine biosynthesis. Am J Clin Nutr 54, Suppl. 6, 1147S-1152S.

44. Rebec GV \& Pierce RC (1994) A vitamin as neuromodulator: ascorbate release into the extracellular fluid of the brain regulates dopaminergic and glutamatergic transmission. Prog Neurobiol 43, 537-565.

45. Levin EY, Levenberg B \& Kaufman S (1960) The enzymatic conversion of 3,4-dihydroxyphenylethylamine to norepinephrine. J Biol Chem 235, 2080-2086.

46. Hoehn SK \& Kanfer JN (1980) Effects of chronic ascorbic acid deficiency on guinea pig lysosomal hydrolase activities. J Nutr 110, 2085-2094.

47. Deana R, Bharaj BS, Verjee ZH, et al. (1975) Changes relevant to catecholamine metabolism in liver and brain of ascorbic acid deficient guinea-pigs. Int J Vitam Nutr Res 45, 175-182.

48. Dremencov E, el Mansari M \& Blier P (2009) Brain norepinephrine system as a target for antidepressant and mood stabilizing medications. Curr Drug Targets 10, 1061-1068.

49. May JM, Qu ZC \& Meredith ME (2012) Mechanisms of ascorbic acid stimulation of norepinephrine synthesis in neuronal cells. Biochem Biophys Res Commun 426, 148-152.

50. Englard S \& Seifter S (1986) The biochemical functions of ascorbic acid. Annu Rev Nutr 6, 365-406.

51. Eipper BA, Mains RE \& Glembotski CC (1983) Identification in pituitary tissue of a peptide $\alpha$-amidation activity that acts on glycine-extended peptides and requires molecular oxygen, copper, and ascorbic acid. Proc Natl Acad Sci U S A 80, 5144-5148.

52. Luck MR \& Jungclas B (1987) Catecholamines and ascorbic acid as stimulators of bovine ovarian oxytocin secretion. J Endocrinol 114, 423-430.

53. Ishak WW, Kahloon M \& Fakhry H (2011) Oxytocin role in enhancing well-being: a literature review. J Affect Disord 130, 1-9.

54. Owen AJ, Batterham MJ, Probst YC, et al. (2005) Low plasma vitamin E levels in major depression: diet or disease? Eur J Clin Nutr 59, 304-306.

55. Maes M, De Vos N, Pioli R, et al. (2000) Lower serum vitamin E concentrations in major depression. Another marker of lowered antioxidant defenses in that illness. J Affect Disord 58, 241-246.

56. Tanaka K, Hashimoto T, Tokumaru S, et al. (1997) Interactions between vitamin $\mathrm{C}$ and vitamin $\mathrm{E}$ are observed in tissues of inherently scorbutic rats. J Nutr 127, 2060-2064.

57. Burk RF, Christensen JM, Maguire MJ, et al. (2006) A combined deficiency of vitamins $\mathrm{E}$ and $\mathrm{C}$ causes severe central nervous system damage in guinea pigs. J Nutr 136, 1576-1581.

58. Campbell DR, Gross MD, Martini MC, et al. (1994) Plasma carotenoids as biomarkers of vegetable and fruit intake. Cancer Epidemiol Biomarkers Prev 3, 493-500.

59. Nishiyama I, Fukuda T \& Oota T (2005) Genotypic differences in chlorophyll, lutein, and $\beta$-carotene contents in the fruits of Actinidia species. J Agric Food Chem 53, 6403-6407. 